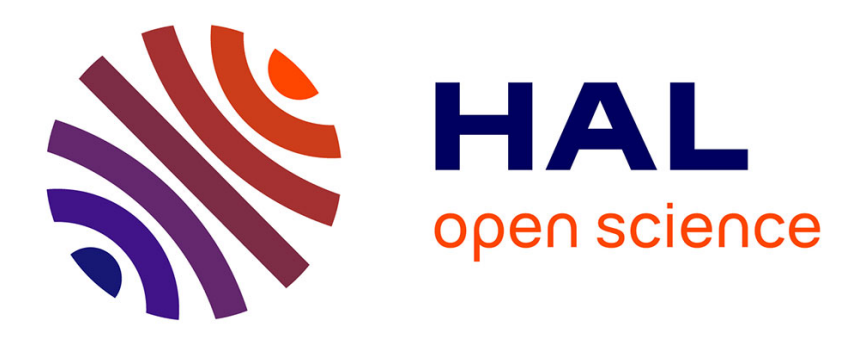

\title{
Dynamics of structural phase transition in a 15-compounds
}

\author{
R. Kragler, H. Thomas
}

\section{To cite this version:}

R. Kragler, H. Thomas. Dynamics of structural phase transition in a 15-compounds. Journal de Physique Lettres, 1975, 36 (5), pp.153-156. 10.1051/jphyslet:01975003605015300 . jpa-00231176

\section{HAL Id: jpa-00231176 https://hal.science/jpa-00231176}

Submitted on 1 Jan 1975

HAL is a multi-disciplinary open access archive for the deposit and dissemination of scientific research documents, whether they are published or not. The documents may come from teaching and research institutions in France or abroad, or from public or private research centers.
L'archive ouverte pluridisciplinaire HAL, est destinée au dépôt et à la diffusion de documents scientifiques de niveau recherche, publiés ou non, émanant des établissements d'enseignement et de recherche français ou étrangers, des laboratoires publics ou privés. 


\title{
DYNAMICS OF STRUCTURAL PHASE TRANSITION IN A 15-COMPOUNDS
}

\author{
R. KRAGLER $\left({ }^{*}\right)$ and H. THOMAS $(* *)$ \\ Institut für Theoretische Physik der Universität Frankfurt, \\ D-6 Frankfurt/M., Germany
}

(Reçu le 20 mai 1974, révisé le 17 février 1975, accepté le 24 février 1975)

\begin{abstract}
Résumé. - On montre que des processus liés à des redistributions électroniques dans la bande $\mathrm{d}$ ont une forte influence sur la dispersion des phonons et le facteur de structure dynamique.

Abstract. - It is shown that electronic d-band redistribution processes have a strong effect on the phonon dispersion and on the dynamic structure factor.
\end{abstract}

The cubic-to-tetragonal phase transitions occurring in A 15-compounds are associated with a softening of the transverse acoustic (TA) $\varepsilon_{x x}-\varepsilon_{y y}$ shear mode [1-3]. Inelastic neutron scattering experiments $[4,5]$ have shown a substantial softening extending over an appreciable region of the Brillouin zone. In order to account for these experiments, a theoretical model based on the deformation-potential coupling between d-electrons and elastic strain has been investigated in various paper [6-11]. The model has been refined by also considering the coupling to the optical $\Gamma_{12}^{\prime}$ phonon branch into account [12-14]. A different attempt assumes a Kohn anomaly due to a planar Fermi surface $[15,16]$. We adopt the model of Labbé and Friedel $[6,7]$, in which the electronic d-bands are shifted relative to each other by the elastic strain, and for which the phase transition is driven by the energy gain resulting from electronic redistribution processes occuring between the $d$ bands in the distorted phase.

We have studied the influence of these electronic interband relaxation processes, which were not taken into account in previous work [8-14], on the dynamics of the transition. The relaxation frequency $\gamma$ for these interband processes is determined by the overlap of wavefunctions belonging to ions in different chains of the A 15-structure. In contrast, the d-band width $\Delta E$ is determined by the overlap within the chains. Since the interchain overlap will be significantly smaller than the intrachain overlap, one expects $\gamma<\Delta E / \hbar$. Moreover, only interband processes ramain effective in the $q=0$ limit. Therefore, the low-

(*) Present address : Institut für Theoretische Physik, Universität Konstanz, D-775 Konstanz, Germany.

(**) Present address : Institut für Theoretische Physik, Universität Basel, CH-4056 Basel, Switzerland. frequency, long-wavelength behaviour will be dominated by the interband processes considered here.

We find important effects on the temperature dependence of the TA phonon dispersion and on the dynamic structure factor. For the phonon dispersion, we obtain good qualitative agreement with the phonon dispersion results of inelastic neutron scattering $[4,5]$, for an interband relaxation frequency $\gamma$ of the order of $10^{-13} \mathrm{~s}^{-1}$. The assumption of a Kohn anomaly for the interpretation of the results $[15,16]$ appears to be unnecessary. The dynamic structure factor, on the other hand, seems to indicate the presence of an additional, non-electronic relaxation mechanism which is slower by an order of magnitude.

We construct a thermodynamic model in terms of the electronic occupation numbers $\left(n_{x}, n_{y}, n_{z}\right)$ of the three degenerate d-bands, and the components of the elastic strain tensor $\varepsilon$. Disregarding s-d coupling we assume conservation of the total number of d-electrons, $n_{\mathrm{d}}=n_{x}+n_{y}+n_{z}$, such that the electronic state of the system can be described by the two linear combinations

$$
n_{2}=\frac{\left(n_{x}-n_{y}\right)}{\sqrt{2}}, \quad n_{3}=\frac{\left(2 n_{z}-n_{x}-n_{y}\right)}{\sqrt{6}}
$$

of occupation number deviations from the symmetric state $n_{x}=n_{y}=n_{z}=n_{\mathrm{d}} / 3$.

Symmetry considerations show that coupling to the d-bands occurs for the tensor components

$$
\varepsilon_{2}=\frac{\left(\varepsilon_{x x}-\varepsilon_{y y}\right)}{\sqrt{2}}, \quad \varepsilon_{3}=\frac{\left(2 \varepsilon_{z z}-\varepsilon_{x x}-\varepsilon_{y y}\right)}{\sqrt{6}}
$$

spanning the E-representation subspace which contains all orthorhombic and tetragonal distortions 
relevant for the lattice transformation. All other components of the strain tensor are disregarded.

In the cubic phase, the lowest order contributions to the free energy density are the elastic free energy

$$
F_{\mathrm{L}}=\frac{1}{2} C\left(\varepsilon_{2}^{2}+\varepsilon_{3}^{2}\right),
$$

the electronic free energy

$$
F_{\mathrm{e}}=\frac{1}{2} Z^{-1}\left(n_{2}^{2}+n_{3}^{2}\right),
$$

and the coupling of the d-bands to the lattice deformation

$$
F_{\mathrm{e}-\mathrm{L}}=D\left(\varepsilon_{2} n_{2}+\varepsilon_{3} n_{3}\right) .
$$

Here, $C=c_{11}-c_{12}$ is the bare shear modulus in absence of electron-lattice coupling, $D$ is the deformation potential, and $Z=Z(T)$ represents an effective density of states, the temperature dependence of which is determined by the actual form of the d-bands. In A 15-compounds such as $\mathrm{Nb}_{3} \mathrm{Sn}$ and $\mathrm{V}_{3} \mathrm{Si}, Z(T)$ decreases with increasing temperature.

The coupling term (5) gives rise to an induced stress $(\alpha=2,3)$

$$
\sigma_{\alpha}^{\text {ind }}=-\partial F_{\mathrm{e}-\mathrm{L}} / \partial \varepsilon_{\alpha}=-D n_{\alpha},
$$

and an induced electronic potential

$$
\mu_{\alpha}^{\mathrm{ind}}=-\partial F_{\mathrm{e}-\mathrm{L}} / \partial n_{\alpha}=-D \varepsilon_{\alpha} .
$$

In the presence of a static external stress $\sigma_{\alpha}^{\text {ext }}$ there occurs an occupation number deviation

$$
n_{\alpha}=Z \mu_{\alpha}^{\text {ind }}=-Z D \varepsilon_{\alpha},
$$

and the elastic strain is given by the total stress,

$$
C \varepsilon_{\alpha}=\sigma_{\alpha}^{\mathrm{ext}}+\sigma_{\alpha}^{\mathrm{ind}},
$$

with $\sigma_{\alpha}^{\text {ind }}$ determined by eq. (6) and (8). The static response to an external stress is thus described by a renormalized elastic constant

$$
C^{*}(T)=C-Z(T) D^{2} .
$$

This result indicates that the electron-lattice coupling can give rise to a lattice instability for sufficiently high density of states such that $Z(0) D^{2}>C$. The cubic phase will therefore become unstable below a certain temperature $T_{0}$ determined by $C^{*}\left(T_{0}\right)=0$, and it is the TA shear mode belonging to the E-subspace which governs the dynamic lattice instability. The temperature dependence of $Z(T)$ can be taken empirically from measurements of the renormalized elastic constant $C^{*}(T)$ [1-3].

In order to study the dynamics of the model we construct equations of motion for $n_{\alpha}$ and $\varepsilon_{\alpha}$. For the electronic occupation number deviations $n_{\alpha}$ a simple Debye-type relaxation to the instantaneous equilibrium values $n_{\alpha}=Z \mu_{\alpha}^{\text {ind }}$ is assumed,

$$
\dot{n}_{\alpha}=-\gamma\left(n_{\alpha}-Z \mu_{\alpha}^{\text {ind }}\right),
$$

where $\gamma$ is an electronic interband relaxation frequency. Conservation of the number of d-electrons is guaranteed by definition eq. (2).

The elastic deformation u satisfies the usual equations of motion with the induced stress, eq. (10), taken into account

$$
\rho \ddot{\mathbf{u}}=\nabla \cdot\left(C \varepsilon-\sigma^{\text {ind }}\right) .
$$

(Note, that there is no difference between $C^{\text {is }}$ and $C^{\text {ad }}$ for the shear modulus $C=c_{11}-c_{12}$.)

For simplicity, we confine ourselves to the TA shear mode with $\mathbf{q} \|(110)$ and $\mathbf{u} \|(110)$, whence $\varepsilon_{2}=i q u / \sqrt{2}$ and $\varepsilon_{3}=0$. Then, eqs. (14) and (15) reduce to

$$
\begin{gathered}
(\omega+i \gamma) n-(\gamma Z D / \sqrt{2}) q u=0 \\
(i D / \sqrt{2}) q n+\rho\left(\omega^{2}-v_{0}^{2} q^{2}\right) u=0
\end{gathered}
$$

where the free sound velocity $v_{0}$ is given by $v_{0}^{2}=C / 2 \rho$. We further introduce the renormalized velocity by $v^{2}=C^{*} / 2 \rho$. The system of coupled equations, eq. (13), leads to a cubic secular equation,

$$
\left(\omega^{2}-v_{0}^{2} q^{2}\right)(\omega+i \gamma)=-i \gamma\left(v_{0}^{2}-v^{2}\right) q^{2}
$$

the roots of which yield three normal-mode frequencies $\omega=\Omega_{q}^{(i)}(i=1,2,3)$. Since $C^{*}(T)$ and therefore $v^{2}$ are determined by static measurements, the electronic relaxation frequency $\gamma$ is the only adjustable model parameter. The behaviour of the normal mode frequencies $\Omega_{q}^{(i)}$ as function of $q$ is displayed in figures 1 and 2 for three values of $v / v_{0}$. For $q \rightarrow 0$, one obtains renormalized phonon modes $\Omega_{q}= \pm v q$, the velocity of which goes to zero at the stability limit $T=T_{0}$, and an unrenormalized relaxation mode $\Omega=-i \gamma$. With increasing $q$ one obtains at first a damping of the renormalized phonon modes which is given to $0\left(q^{2}\right)$ by $\Omega_{q}^{\prime \prime}=-i v_{0}^{2} q^{2} / 2 \gamma$. For $v / v_{0}<1 / 3$, i.e. for temperatures little above $T_{0}$, $\Omega_{q}^{\prime}$ goes through a maximum and reaches zero at $v_{0} q \sim 2 \gamma v / v_{0}$. Above this value, all three modes are overdamped with purely imaginary frequencies until at $v_{0} q \gtrsim \gamma / 2$ damped but propagating modes reappear which approach the unrenormalized values

$$
\Omega_{q}= \pm v_{0} q \text { at } v_{0} q \gg \gamma / 2,
$$

leaving a renormalized relaxation mode $\Omega \rightarrow-i v^{2} / v_{0}^{2}$. With increasing $v / v_{0}$, the gap where $\Omega_{q}^{\prime}=0$ narrows and finally disappears at $v / v_{0}=1 / 3$ corresponding to $T \simeq 61 \mathrm{~K}$ for $\mathrm{Nb}_{3} \mathrm{Sn}$. For higher temperatures where $v / v_{0}>1 / 3$, the modes remain propagating for all $q$. Right at the stability limit $T_{0}$ where $v=0$, we obtain exact roots of eq. (14)

$$
\Omega_{q}^{(1)}=0 \quad \Omega_{q}^{(2,3)}= \pm \sqrt{v_{0}^{2} q^{2}-\gamma^{2} / 4}-i \gamma / 2
$$

showing a gap with $\Omega_{q}^{\prime(2,3)}=0$ between $q=0$ and $q=\gamma / 2 v_{0}$. 

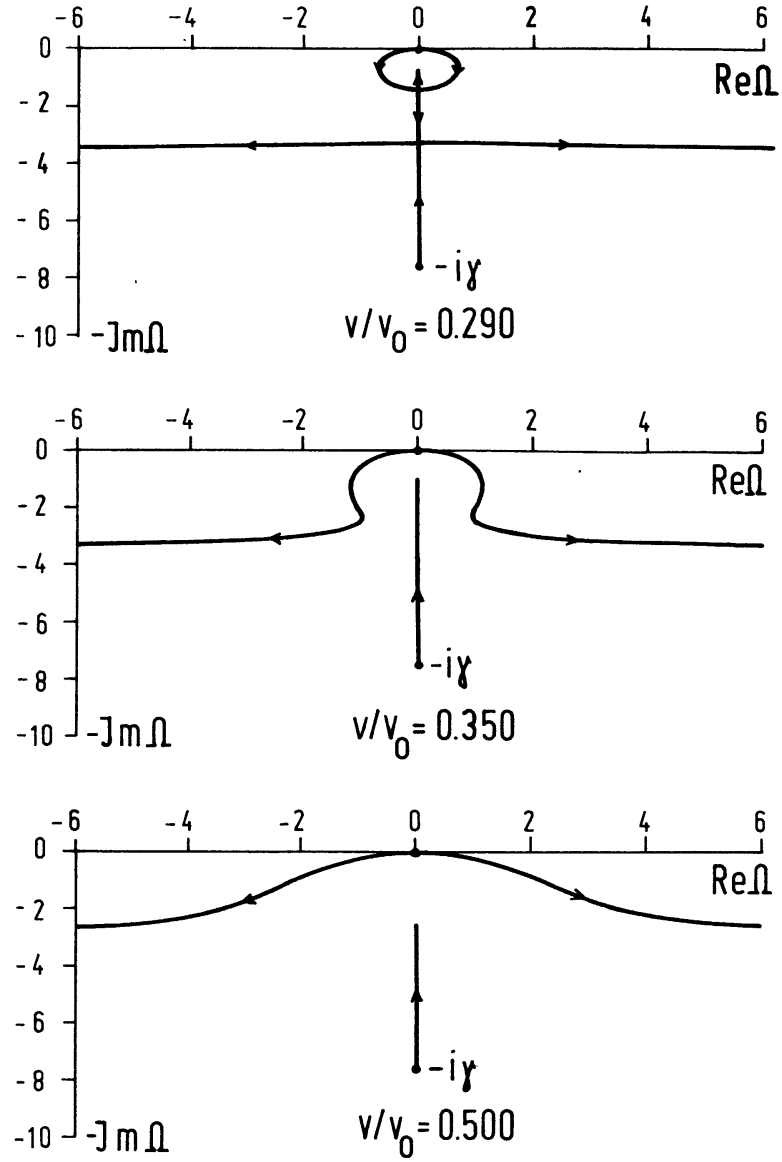

Fig. 1. - Motion of the coupled-mode frequencies in the complex frequency plane with wave-vector $q=0$ to $q=q_{B Z} / 2$. Frequency scale in $\mathrm{meV}\left(\mathrm{Nb}_{3} \mathrm{Sn}\right)$.

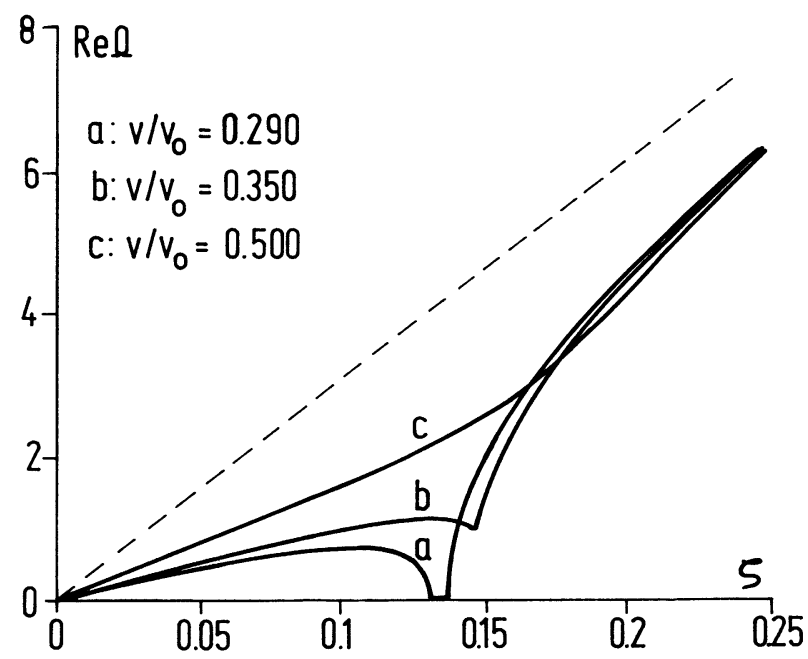

Fig. 2. - Dispersion of the real part of the $(110,1 \overline{1} 0)$ mode frequency for the three temperatures $\left(\mathrm{Nb}_{3} \mathrm{Sn}\right)$.

We obtain good qualitative agreement of our calculation with phonon dispersion measured by Axe and Shirane [4, 5] on $\mathrm{Nb}_{3} \mathrm{Sn}$, if we choose $\gamma / \omega_{0}=0.250$ where $\omega_{0}=30.7 \mathrm{meV}$ is the extrapolated phonon energy for $\zeta \equiv q / 2 q_{B z}=1$. This corresponds to an electronic relaxation frequency of $\gamma=10^{13} \mathrm{~s}^{-1}$. This rather low value of $\gamma$ reflects the small overlap of the wave functions belonging to different bands.

The results of inelastic neutron scattering experiments (energy transfer $\omega$, momentum transfer $\mathbf{Q}$ ) are described in terms of the dynamic structure factor

$$
S(\mathbf{Q}, \omega) \propto \sum_{i, j} Q_{i} Q_{j} S_{i j}(\mathbf{q}, \omega)
$$

where $S_{i j}(\mathbf{q}, \omega)$ is the Fourier transform of the displacement correlation function $\left\langle u_{i}(\mathbf{x} t) u_{j}\left(\mathbf{x}^{\prime} 0\right)\right\rangle$ at $\mathbf{q}=\mathbf{Q}-\mathbf{K}$ in the first Brillouin zone. The correlations of the TA mode $\mathbf{q}\|(110), \mathbf{u}\|(1 \overline{1} 0)$ considered above are thus given by the scattering with $\mathbf{Q} \|(110)$ such that $S(\mathbf{Q}, \omega)$ is proportional to $S_{x x}-S_{x y}$. This quantity can be obtained via the fluctuationdissipation theorem $\left(\hbar \omega \ll k_{\mathrm{B}} T\right)$

$$
\begin{aligned}
& S_{x x}(q, \omega)-S_{x y}(q, \omega)= \\
& =\left(2 k_{\mathbf{B}} T / \hbar \omega\right) \operatorname{Im}\left[\chi_{x x}(q, \omega)-\chi_{x y}(q, \omega)\right]
\end{aligned}
$$

from the displacement response tensor $\chi(q, \omega)$ which describes the response to an external force. Adding a dynamic perturbation to the eq. of motion (12) and solving the inhomogeneous system, one finds

$$
\begin{aligned}
& \chi_{x x}(q, \omega)-\chi_{x y}(q, \omega)= \\
& \quad=(1 / \rho)\left[\left(v_{0}^{2} q^{2}-\omega^{2}\right)-i \gamma\left(v_{0}^{2}-v^{2}\right) q^{2} /(\omega+i \gamma)\right]^{-1}
\end{aligned}
$$

which yields a structure factor of the same form as given by Shirane and Axe $[4,5]$. This result gives rise to the following behaviour, displayed in figure 3 as function of $q$. For $v_{0} q<\sqrt{2} \gamma v / v_{0}$, we find two phonon peaks near the renormalized phonon frequencies $\omega= \pm v q$. For $v / v_{0}<1 / 2$, these two peaks merge into a central peak at $v_{0} q=\sqrt{2} \gamma v / v_{0}$. At still higher $q$-values $v_{0} q \geqslant \gamma$, two new side bands appear near the unrenormalized phonon frequencies $\omega= \pm v_{0} q$, in addition to the central component.

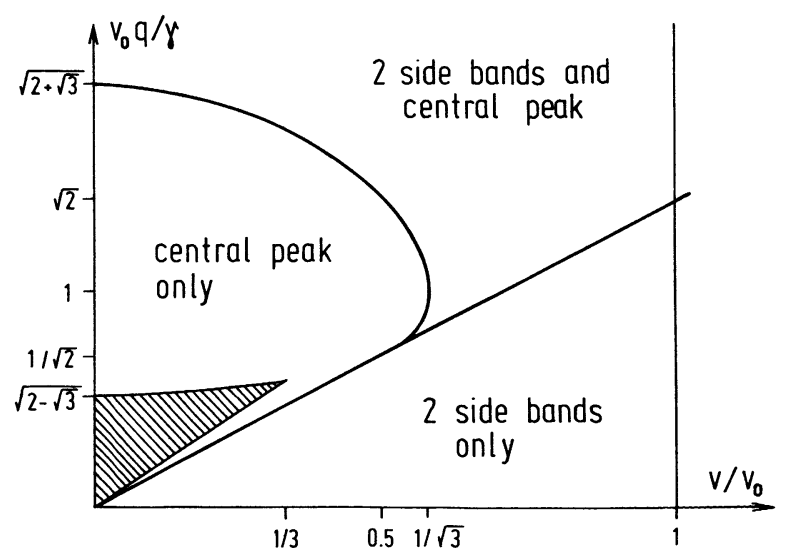

FIG. 3. - Behaviour of the dynamic structure factor as function of $q$ and $v / v_{0}$. In the hatched area exist three overdamped modes, everywhere else exist two propagating modes and one relaxation mode. 
For higher temperatures, $v / v_{0}>1 / 2$, the two-peak structure changes directly into the three-peak structure at $v_{0} q=\sqrt{2} \gamma v / v_{0}$.

However, these results do not explain the threepeak structure factor observed by Shirane and Axe at very small $q$-values. In order to reproduce the observed line shape, it would be necessary to choose the relaxation frequency $\gamma$ smaller by an order of magnitude than found above from the phonon dispersion. This inconsistency seems to indicate that the behaviour at very low frequencies is governed by an additional, non-electronic relaxation mechanism with a relaxation frequency of the order $10^{11} \mathrm{~s}^{-1}$. It has been suggested by Shirane and Axe $[4,5]$ that this relaxation is due to the transition from collisiondominated to collision-free phonon propagation [17, 18].

In order to obtain a description of the tetragonal phase, one has to include third and fourth order terms in the electronic energy, eq. (4). One then finds a new equilibrium state with spontaneous strain $\varepsilon_{3}$ coupled with a non-zero $n_{3}$. It is straightforward to extend our treatment to the study of the dynamical behaviour in this phase.

\section{References}

[1] Testardi, L. R. and Bateman, T. B., Phys. Rev. 154 (1967) 402.

[2] Keller, K. R. and Hanak, J. J., Phys. Rev. 154 (1967) 628.

[3] Rehwald, W., Rayl, M., Cohen, R. W. and Cody, G. D., Phys. Rev. B 6 (1972) 363.

[4] Shirane, G. and Axe, J. D., Phys. Rev. B 4 (1971) 2957; Phys. Rev. Lett. 27 (1971) 1803.

[5] Axe, J. D. and Shirane, G., Phys. Rev. B 8 (1973) 1965.

[6] LabBé, J. and Friedel, J., J. Physique 27 (1966) 153; 27 (1966) 303.

[7] Barišić, S. and Labbé, J., J. Phys. Chem. Solids 28 (1967) 2477.

[8] Cohen, R. W., Cody, G. D. and Halloran, J. J., Phys. Rev. Lett. 19 (1967) 840.
[9] Pytte, E., Phys. Rev. B 4 (1971) 1094.

[10] Dieterich, W. and Klose, W., Z. Phys. 246 (1971) 323.

[11] Dieterich, W. and Schuster, H., Phys. Lett. 35A (1971) 48.

[12] Klein, B. M. and Birman, J. L., Phys. Rev. Lett. 25A (1970) 1014.

[13] Sham, L. J., Phys. Rev. B 6 (1972) 3584.

[14] Noolandi, J. and Sham, L. J., Phys. Rev. B 8 (1973) 2468

[15] BARIŠIĆ, S., Solid State Commun. 9 (1971) 1507.

[16] Horovitz, B., Weger, M., Gutfreund, M., Phys. Rev. B 9 (1974) 1246.

[17] ENZ, C. P., to be published.

[18] KLEIN, R., in Anharmonic Lattices, Structural Transitions and Melting, Ed. by T. Riste (Noordhoff, Leiden) 1974. 